\title{
PREVALÊNCIA E FATORES ASSOCIADOS À OCORRÊNCIA DE LESÕES DURANTE A PRÁTICA DE ATIVIDADE FÍSICA
}

\author{
PREVALENCE AND FACTORS ASSOCIATED WITH INJURIES DURING LEISURE-TIME PHYSICAL \\ ACTIVITY PRACTICE
}

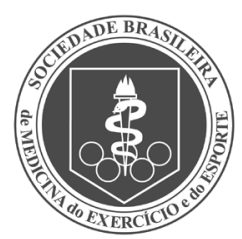

Artigo Original

\author{
PREVALENCIA Y FACTORES ASOCIADOS A LA OCURRENCIA DE LESIONES DURANTE LA \\ PRÁCTICA DE LA ACTIVIDAD FÍSICA
}

Airton José Rombaldi'

(Educador Físico)

Marcelo Cozzensa da Silva'

(Educador Físico)

Maurício Tavares Barbosa ${ }^{3}$

(Fisioterapeuta)

Ricardo Corrêa Pinto ${ }^{3}$

(Fisioterapeuta)

Mario Renato Azevedo ${ }^{1}$

(Educador Físico)

Pedro Curi Hallal'

(Educador Físico)

Fernando Vinholes Siqueira ${ }^{2}$

(Educador Físico e Fisioterapeuta)

1. Escola Superior de Educação

Física, Universidade Federal de

Pelotas, Pelotas, RS, Brasil.

2. Faculdade de Terapia Ocupacional,

Universidade Federal de Pelotas,

Pelotas RS, Brasil.

3. Faculdade de Fisioterapia,

Universidade Católica de Pelotas,

Pelotas, RS, Brasil.

\section{Correspondência:}

PPG em Educação Física, Universidade

Federal de Pelotas, Rua Luis de

Camões 625, Pelotas, RS, Brasil.

96055-630.

rombaldi@brturbo.com.br

\section{RESUMO}

Introdução: Embora estudos demonstrem a importância da atividade física na prevenção e manutenção de um padrão de vida saudável, a prática pode determinar um aumento no risco da ocorrência de lesões entre aqueles praticantes de atividades desportivas. Objetivo: Determinar a prevalência e os fatores associados à ocorrência de lesões durante a prática de atividade física no tempo de lazer na cidade de Pelotas, RS, Brasil. Métodos: Estudo transversal de base populacional realizado na área urbana do município de Pelotas, RS, Brasil. Uma amostra levando em consideração os diversos conglomerados foi selecionada. Características sociodemográficas, econômicas, comportamentais, nutricionais e relacionadas à ocorrência de lesões durante a prática de atividade física foram coletadas por meio de questionário padronizado e pré-codificado. Resultados: O percentual de lesões em sujeitos praticantes de atividade física no lazer foi de 21,9\% (IC95\% 18,3 - 25,9). As lesões mais prevalentes foram contusões $(39,6 \%)$, luxações $(30,2 \%)$ e distensões $(11,3 \%)$ e a prática de futebol e musculação foram as atividades onde mais ocorreram lesões. As lesões estiveram associadas ao sexo masculino, idades mais jovens e a indivíduos de cor da pele preta e parda. Conclusão: Embora a prevalência de lesões entre praticantes seja importante, os benefícios decorrentes de um estilo de vida ativo superam os riscos.

Palavras-chave: atividade motora, morbidade, ferimentos e lesões, epidemiologia.

\begin{abstract}
Introduction: Although studies show the importance of physical activity in prevention and maintaining a healthy standard of living, its practice can determine an increased risk of occurrence of injuries among people who practice sports activities. Objective: Determine the prevalence and factors associated with injuries during leisure-time physical activity practice in the city of Pelotas, RS, Brazil. Methods: Population-based cross-sectional study conducted in the urban area of Pelotas, southern Brazil. A sample taking into account the different clusters was selected. Sociodemographic, economic, behavioral, nutritional and physical activity injury related characteristics were collected using a standardized and pre-coded questionnaire. Results: The prevalence of injuries in subjects physically active during leisure time was $21.9 \%$ (C195\% 18.3 - 25.9). The most prevalent injuries were contusions (39.6\%), dislocations (30.2\%) and sprains (11.3\%) and soccer practice and weightlifting were activities where more injuries occurred. The injuries were associated with male gender, younger age and black and mixed race. Conclusion: Although the prevalence of injuries among practitioners is important, the benefits of an active lifestyle outweigh the risks.
\end{abstract}

Keywords: motor activity, morbidity, injuries, epidemiology.

\section{RESUMEN}

Introducción: Aunque estudios demuestren la importancia de la actividad física en la prevención y mantenimiento de un estándar de vida saludable, la práctica puede determinar un aumento en el riesgo de la ocurrencia de lesiones entre aquellos practicantes de actividades deportivas. Objetivo: Determinar la prevalencia y los factores asociados a la ocurrencia de lesiones durante la práctica de actividad física en el tiempo libre en la ciudad de Pelotas, RS, Brazil. Métodos: Estudio transversal de base poblacional realizado en el área urbana del municipio de Pelotas, RS, Brazil. Fue seleccionada una muestra llevando en consideración los diversos conglomerados. Fueron colectadas características sociodemográficas, económicas, comportamentales, nutricionales y relacionadas a la ocurrencia de lesiones durante la práctica de actividad física por medio de cuestionario estandarizado e precodificado. Resultados: El porcentual de lesiones en sujetos practicantes de actividad física en su tiempo libre fue de 21,9\% (IC95\% 18,3-25,9). Las lesiones más prevalentes fueron contusiones (39,6\%), luxaciones (30,2\%) y distensiones (11,3\%) y la práctica de fútbol y musculación fueron las actividades en donde más ocurrieron lesiones. Las lesiones estuvieron asociadas al sexo masculino, edades más jóvenes y a individuos con color de piel negra y parda. Conclusión: Aunque la prevalencia de lesiones entre practicantes sea importante, los beneficios provenientes de un estilo de vida activo superan los riesgos.

Palabras clave: actividad motora, morbidad, heridas y traumatismos, epidemiologia. 


\section{INTRODUÇÃO}

Atualmente a prática regular de atividade física (AF) é uma das melhores maneiras de promover a saúde e prevenir doenças ${ }^{1,2}$. A relação entre atividade física e saúde parece estar intimamente ligada, pois se torna evidente que a atividade física contribui positivamente para a saúde de indivíduos e comunidades, e essa relação vem dando suporte a iniciativas e estratégias adotadas pelo poder público que visam engajar populações em um estilo de vida ativo ${ }^{3,4}$.

Embora estudos demonstrem a importância da atividade física na prevenção e manutenção de um padrão de vida saudável, sua prática pode determinar um aumento no risco da ocorrência de lesões nesse grupo de pessoas ${ }^{5}$, especialmente entre aqueles praticantes de atividades desportivas ${ }^{6,7}$. Estas lesões podem ser determinantes não somente para o afastamento da prática da atividade física como também da atividade profissional, representando prejuízos econômicos importantes, além da necessidade de buscar atendimento especializado $0^{8,9}$.

Estudo realizado na Holanda relatou que um total 2,9 milhões de lesões relacionadas ao esporte aconteceu por ano, sendo que 1,1 milhão (38\%) recebeu atendimento especializado ${ }^{10}$. Na Austrália, estimou-se no ano de 2000 um gasto de um bilhão de dólares devido à lesões relacionadas com a prática de atividade física. Além disso, o estudo reporta que um a cada 17 australianos sofreu alguma lesão suficiente para o afastamento da prática esportiva e/ou para receber atendimento nos serviços de saúde ${ }^{11}$.

Estudos relacionados a esse tema apresentam amostras limitadas e focados fundamentalmente em atletas ou praticantes de espor$t^{12-15}$. Considerando estudos de base populacional, encontramos no Brasil apenas uma publicação, a qual relatou somente o uso de serviço de fisioterapia sem relação com a prática de atividade física ou esporte ${ }^{16}$. Nesse sentido, o objetivo desse estudo foi determinar a prevalência e os fatores associados à ocorrência de lesões durante a prática de atividade física no tempo de lazer na cidade de Pelotas, RS.

\section{MÉTODOS}

Estudo transversal de base populacional com objetivo de estudar diversos desfechos em saúde e AF foi realizado na zona urbana do município de Pelotas, RS, Brasil, cidade com aproximadamente 340 mil habitantes. O Instituto Brasileiro de Geografia e Estatística divide a cidade em 408 setores censitários urbanos domiciliares, ordenados no formato "espiral", do centro para os bairros, dentro dos quais foram selecionados 40 setores de forma sistemática para compor a amostra estudada. Em cada setor foi sorteado aleatoriamente um ponto de partida para a escolha das residências a serem estudadas, ponto a partir do qual visitou-se sistematicamente 15 domicílios por setor, totalizando 600 domicílios. Todos os indivíduos entre 20 e 69 anos residentes nos domicílios sorteados foram entrevistados, com exceção dos que apresentavam incapacidade mental ou física severa que os impedisse de responder ao questionário.

Foram realizados dois cálculos para tamanho amostral. Inicialmente, foi estimada a amostra necessária para avaliar a prevalência de lesões: prevalência estimada de 30,0\% para lesões em atividades físicas de lazer, margem de erro aceitável de 3,0 pontos percentuais, nível de confiança de $95 \%$ e acréscimo de 10\% para perdas e recusas, resultou em uma amostra de 985 sujeitos. Para explorar a associação entre lesões em atividades físicas de lazer e variáveis independentes, os seguintes parâmetros foram utilizados: nível de confiança de $95 \%$, poder de 80\%, relação exposto/não exposto de 1/4 (nível econômico), prevalência de exposição variando de 10\% a 90\%, razão de prevalências de 2,0, acréscimo de 10\% para não respondentes, 15\% para controle de fatores de confusão e efeito de delineamento amostral de
1,5. Com a variação dos parâmetros de prevalência, o maior tamanho amostral encontrado foi de 788 sujeitos.

Características sociodemográficas, econômicas, comportamentais e nutricionais foram avaliadas pela aplicação de um questionário padronizado. Foram coletadas as variáveis sexo (masculino ou feminino), idade em anos (posteriormente categorizada de 20 a 34; 35 a 49; 50 a 69 anos), cor da pele (branca, preta, parda), situação conjugal (casado/vive com companheiro, solteiro, separado, viúvo), nível econômico (determinado segundo classificação da Associação Brasileira de Empresas de Pesquisa) ${ }^{17}$, tabagismo (ex-fumantes, fumantes atuais e nunca fumantes) e estado nutricional (determinado pelo índice de massa corporal - IMC, calculado a partir do peso e estatura autorreferidos e classificado segundo critérios da OMS $)^{18}$. Adicionalmente, foi perguntado aos que relataram a ocorrência de lesão, o tipo de lesão, em que atividade física a mesma aconteceu e a busca por serviço de fisioterapia.

Para determinação do desfecho foram utilizadas duas perguntas. A primeira buscou saber se o indivíduo praticava atividade física no tempo de lazer (O(A) Sr.(a) pratica atividade física no seu tempo livre?). Independente do tipo e do tempo de prática, para aqueles que responderam positivamente a primeira pergunta, foi realizada uma segunda questão: "Alguma vez o(a) Sr.(a) já se machucou praticando uma dessas atividades físicas mencionadas?". Em caso de resposta positiva, o indivíduo foi considerado como tendo o desfecho.

As entrevistas foram realizadas por 20 entrevistadores, os quais possuíam no mínimo 18 anos e ensino médio completo e que passaram por um período de treinamento de 20 horas. Os questionários foram revisados por supervisores, que estavam em contato permanente com os entrevistadores durante o trabalho de campo. O controle de qualidade foi realizado mediante revisitas a 25\% da amostra.

Os questionários, depois de revisados e codificados, foram duplamente digitados utilizando o software EPI-INFO versão 6, com checagem automática de amplitude e consistência e a análise dos dados realizada no programa STATA 12. Foi realizada uma análise descritiva para caracterizar a amostra, e posteriormente análises bivariadas e multivariável. Para todos os testes de hipóteses foi adotado um nível de significância de 5\%.

A análise bivariada verificou a relação entre desfecho e variáveis independentes por meio dos testes de qui-quadrado para heterogeneidade e tendência linear. A análise multivariável foi realizada por meio de regressão de Poisson, com variância robusta e, para controle de confusão, sendo mantidas na análise todas aquelas variáveis que apresentaram valor $p \leq 0,2$ na análise bruta. Todas as análises levaram em consideração o efeito do delineamento amostral, pelo grupo de comandos "svy" do STATA. Na análise multivariável, todas as variáveis foram colocadas ao mesmo tempo e a seguir realizou-se seleção para trás.

O projeto foi aprovado pelo Comitê de Ética em Pesquisa da Escola Superior de Educação Física da Universidade Federal de Pelotas sob protocolo número 005/2008 e os dados foram coletados após consentimento informado dos sujeitos. A confidencialidade das informações e o direito de recusa em participar foram plenamente garantidos.

\section{RESULTADOS}

Em 514 domicílios sorteados com moradores adultos, 1.062 eram elegíveis. Destes, 972 responderam ao questionário (taxa de não-respondentes de 8,5\%). A variável independente com maior número de valores ignorados foi o IMC, sendo que 9\% (87 indivíduos) não souberam informar sua massa corporal e/ou estatura.

A amostra foi constituída de aproximadamente $36 \%$ de pessoas com idade entre 35 a 49 anos, sendo que a média de idade foi de 41 anos $( \pm 13,4)$. A maior parte da amostra estudada (57\%) era do sexo 
feminino, de cor da pele branca (82,4\%) e níveis econômicos C/D/E (59,9\%). Em relação a situação conjugal, cerca de $63 \%$ eram casados ou viviam com companheiro(a). Um total de $49,4 \%$ dos indivíduos nunca havia fumado e mais da metade $(50,8 \%)$ apresentava sobrepeso/obesidade (tabela 1).

Da totalidade dos indivíduos entrevistados, 50,9\% (475) relataram praticar algum tipo de atividade física no tempo de lazer. Dentre as 47 diferentes atividades mencionadas, caminhada $(42,6 \%)$, futebol $(21,5 \%)$, pedalar $(18,6 \%)$, musculação $(6,5 \%)$ e corrida $(5,5 \%)$ foram as mais prevalentes. A figura 1 mostra as atividades praticadas no lazer, estratificadas por sexo. Para o sexo masculino, o futebol $(39,1 \%)$ e a caminhada $(30,2 \%)$ foram as atividades físicas mais relatadas e, entre as mulheres, a cami-

Tabela 1. Características da amostra total e de indivíduos que declararam praticar atividade física no tempo de lazer da cidade de Pelotas ( $n=972$ ).

\begin{tabular}{|c|c|c|c|c|}
\hline \multirow{2}{*}{ Variáveis } & \multicolumn{2}{|c|}{ Faziam atividade física } & \multicolumn{2}{|c|}{ Total } \\
\hline & $\mathrm{N}$ & $\%$ & $\mathrm{~N}$ & (\%) \\
\hline \multicolumn{5}{|l|}{ Sexo } \\
\hline Masculino & 245 & 51,6 & 418 & 43,0 \\
\hline Feminino & 230 & 48,4 & 554 & 57,0 \\
\hline \multicolumn{5}{|l|}{ Idade (anos) } \\
\hline 20 a 34 & 189 & 39,8 & 347 & 35,7 \\
\hline 35 a 49 & 158 & 33,2 & 349 & 35,9 \\
\hline 50 a 69 & 128 & 27,0 & 276 & 28,4 \\
\hline \multicolumn{5}{|l|}{ Cor da Pele } \\
\hline Branca & 399 & 84,2 & 797 & 82,3 \\
\hline Preta & 51 & 10,8 & 116 & 12,0 \\
\hline Parda & 24 & 5,0 & 55 & 5,7 \\
\hline \multicolumn{5}{|l|}{$\begin{array}{c}\text { Nível } \\
\text { Econômico } \\
\text { ABEP* }\end{array}$} \\
\hline A & 64 & 13,8 & 84 & 8,8 \\
\hline B & 159 & 34,2 & 298 & 31,3 \\
\hline C & 166 & 35,7 & 395 & 41,5 \\
\hline$D / E$ & 76 & 16,3 & 175 & 18,4 \\
\hline \multicolumn{5}{|l|}{$\begin{array}{l}\text { Situação } \\
\text { Conjugal }\end{array}$} \\
\hline $\begin{array}{l}\text { Casado / com } \\
\text { companheiro }\end{array}$ & 286 & 60,2 & 615 & 63,3 \\
\hline Solteiro & 129 & 27,2 & 229 & 23,5 \\
\hline Separado & 38 & 8,0 & 74 & 7,6 \\
\hline Viúvo & 22 & 4,6 & 54 & 5,6 \\
\hline \multicolumn{5}{|l|}{ Tabagismo } \\
\hline Nunca fumou & 259 & 54,5 & 480 & 49,4 \\
\hline Fumante & 117 & 24,6 & 276 & 28,4 \\
\hline Já Fumou & 99 & 20,9 & 216 & 22,2 \\
\hline \multicolumn{5}{|l|}{$\begin{array}{c}\text { IMC } \\
\left(\mathrm{Kg} / \mathrm{m}^{2}\right) \# \&\end{array}$} \\
\hline Normal & 218 & 49,2 & 431 & 48,7 \\
\hline Sobrepeso & 170 & 38,4 & 321 & 36,3 \\
\hline Obeso & 55 & 12,4 & 133 & 15,0 \\
\hline
\end{tabular}

*Associação Brasileira de Empresas de Pesquisa; \#índice de massa corporal; \&Variável com maior númer de missings.

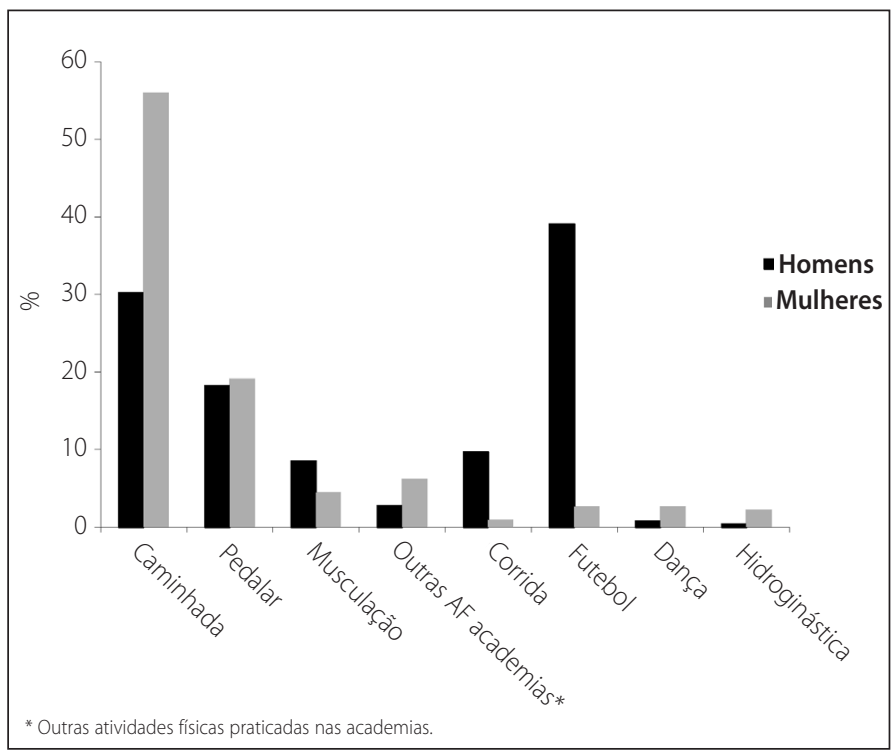

Figura 1. Frequência e tipo de atividades físicas praticadas no lazer pelos sujeitos da amostra $(n=475)$.

nhada $(56,0 \%)$ e pedalar $(19,1)$ apareceram como as mais frequentes.

Entre os praticantes de atividades físicas no tempo de lazer, o percentual de lesões decorrentes da prática foi de 21,9\% (IC 95\% $_{18,3}$ - 25,9) (104 indivíduos). Um total de 52 diferentes lesões foi relatado. As maiores prevalências de lesões foram para contusões (39,6\%), luxações (30,2\%), distensões (11,3\%) e tendinites (3,8\%). A figura 2 mostra a frequência de lesões por tipo de atividade física relatada. Futebol e musculação foram as atividades onde mais ocorreram lesões nos respondentes ( $54 \%$ e 19,4\%, respectivamente). Entre os indivíduos que se lesionaram praticando atividades físicas, 28\% procuraram atendimento fisioterápico.

A tabela 2 descreve a prevalência de lesões e fatores associados segundo variáveis independentes em estudo. Maiores prevalências de lesão foram encontradas em indivíduos do sexo masculino, mais jovens e entre indivíduos de cor de pele preta e parda. As análises bivariada e multivariável identificaram as mesmas variáveis associadas ao desfecho em estudo. Em relação a análise multivariável, indivíduos do sexo feminino apresentaram proteção de 50\% em relação aos seus pares. À medida que a idade avançou, menor o risco de sofrer lesões relacionadas a prática de atividades físicas no lazer, sendo que o grupo na faixa etária de 50 a 69 anos apresentou uma proteção de 40\% em relação ao grupo mais jovem (20 a 34 anos). Pessoas de cor da pele preta e parda apresentaram respectivamente $110 \%$ e $90 \%$ mais risco de lesões em consequência da prática quando comparados ao de cor da pele branca.

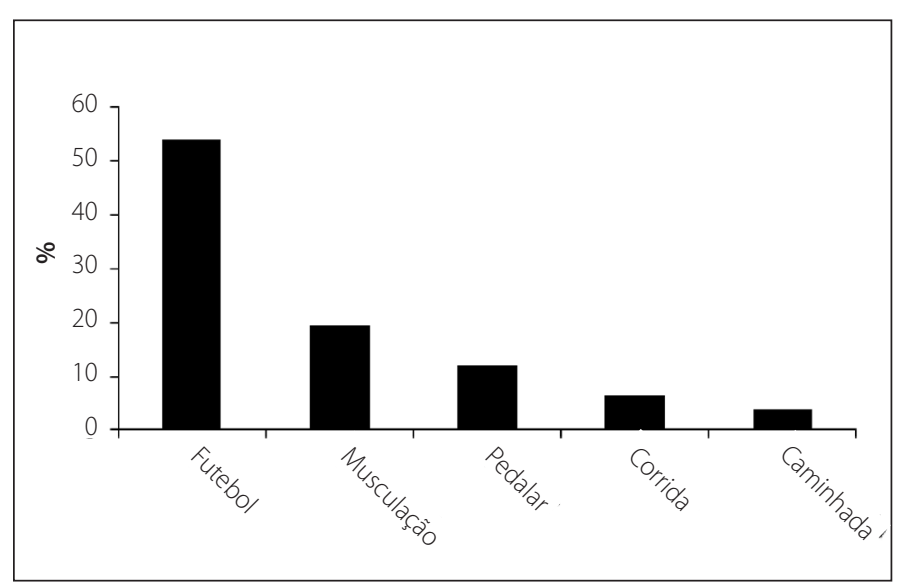

Figura 2. Frequência de lesões por tipo de atividade física relatada ( $n=475)$. 
Tabela 2. Prevalência de lesões e fatores associados entre indivíduos fisicamente ativos no lazer da cidade de Pelotas/RS.

\begin{tabular}{|c|c|c|c|c|}
\hline Variáveis & Prevalência & $\begin{array}{l}\text { RP bruta } \\
\text { (IC95\%) }\end{array}$ & $\begin{array}{l}\text { RP ajustada } \\
\text { (IC95\%)* }\end{array}$ & Valor $p$ \\
\hline Sexo & & & & $<0,001$ \\
\hline Masculino & $30,6 \%$ & 1,0 & 1,0 & \\
\hline Feminino & $12,6 \%$ & $0,4(0,3-0,6)$ & $0,5(0,3-0,7)$ & \\
\hline Idade (anos) & $27,0 \%$ & & & $0,02^{\top}$ \\
\hline $20-34$ & $22,1 \%$ & 1,0 & 1,0 & \\
\hline $35-49$ & $14,1 \%$ & $0,8(0,6-1,2)$ & $0,9(0,6-1,3)$ & \\
\hline $50-69$ & & $0,5(0,3-0,8)$ & $0,6(0,4-0,9)$ & \\
\hline Cor da Pele & $18,8 \%$ & & & $<0,001$ \\
\hline Branca & $39,2 \%$ & 1,0 & 1,0 & \\
\hline Preta & $37,5 \%$ & $2,1(1,4-3,1)$ & $2,1(1,4-3,1)$ & \\
\hline Parda & & $2,0(1,1-3,5)$ & $1,9(1,1-3,2)$ & \\
\hline $\begin{array}{l}\text { Nível } \\
\text { Econômico } \\
\text { ABEP }\end{array}$ & $28,1 \%$ & & & $0,1^{\top}$ \\
\hline A & $23,3 \%$ & 1,0 & 1,0 & \\
\hline B & $18,1 \%$ & $0,8(0,5-1,4)$ & $0,9(0,5-1,4)$ & \\
\hline C & $22,4 \%$ & $1,0(0,6-1,7)$ & $1,1(0,7-1,9)$ & \\
\hline $\mathrm{D} / \mathrm{E}$ & & $1,3(0,7-2,2)$ & $1,4(0,8-2,6)$ & \\
\hline $\begin{array}{l}\text { Situação } \\
\text { Conjugal }\end{array}$ & $19,9 \%$ & & & 0,3 \\
\hline $\begin{array}{c}\text { Casado/ } \\
\text { companheiro }\end{array}$ & $28,7 \%$ & 1,0 & 1,0 & \\
\hline Solteiro & $15,9 \%$ & $1,4(1,0-2,1)$ & $1,2(0,8-1,7)$ & \\
\hline Separado & $18,2 \%$ & $0,8(0,4-1,7)$ & $1,0(0,5-2,0)$ & \\
\hline Viúvo & & $0,9(0,4-2,3)$ & $1,9(0,7-5,0)$ & \\
\hline Tabagismo & $20,9 \%$ & & & 0,8 \\
\hline Nunca fumou & $28,2 \%$ & 1,0 & 1,0 & \\
\hline Fumante & $17,2 \%$ & $1,4(0,9-2,0)$ & $1,2(0,8-1,7)$ & \\
\hline Já fumou & & $0,8(0,5-1,3)$ & $0,9(0,5-1,4)$ & \\
\hline $\operatorname{IMC}\left(\mathrm{Kg} / \mathrm{m}^{2}\right)^{\# \&}$ & $24,3 \%$ & & & 0,4 \\
\hline Normal & $20,0 \%$ & 1,0 & 1,0 & \\
\hline Sobrepeso & $16,4 \%$ & $0,8(0,6-1,2)$ & $0,9(0,6-1,4)$ & \\
\hline Obeso & & $0,7(0,4-1,3)$ & $0,8(0,4-1,4)$ & \\
\hline
\end{tabular}

Teste de Wald para tendência linear; \# Índice de massa corporal; \& Variável com maior número de missings; * Todas as variáveis foram ajustadas entre si (ajustada também para a variável tipo de atividade física praticada).

\section{DISCUSSÃO}

Em uma amostra representativa da cidade de Pelotas, a prevalência de lesões entre praticantes de atividade física no lazer foi de 21,9\%. Nosso resultado foi similar ao encontrado em um estudo realizado na Holanda onde a prevalência de lesões por atividade esportiva foi de $21,5 \%{ }^{10}$.

O presente estudo mostrou que algumas atividades como futebol, musculação e pedalar, foram as que produziram o maior número de lesões. Apesar das diferenças culturais, um estudo australiano mostrou que essas atividades estão também entre aquelas que mais produziram lesões naquele país ${ }^{19}$. A incidência de determinadas lesões esportivas pode variar como decorrência de uma série de fatores, tais como o tipo de esporte praticado, o tempo da prática esportiva e se a prática, mesmo que recreacional, tem caráter competitivo.
As lesões mais frequentes na amostra estudada foram as contusões, luxações e distensões o que corrobora com os resultados descritos por Conte et al. em estudo realizado em Sorocaba, $\mathrm{SP}^{20}$, o qual encontrou entre as maiores frequências de lesões musculares, os entorses, as contusões e, entre as menos frequentes, as fraturas. A ocorrência de lesões na prática de atividades físicas é comum e a incidência das mesmas pode estar relacionada com diferentes fatores de risco. Existem os fatores de risco intrínsecos congênitos, muitas vezes incontroláveis, e aqueles resultantes de fatores extrínsecos adquiridos por aspectos ambientais ${ }^{21}$.

A associação entre lesões e o sexo masculino encontrada nesse estudo segue a encontrada por Dempsey et al. ${ }^{8}$ e Hootmann et al..$^{22}$ em amostras de indivíduos Norte-americanos. Vários estudos epidemiológicos relataram que indivíduos do sexo masculino eram mais ativos no tempo de lazer e praticavam mais atividades físicas vigorosas quando comparados a seus pares ${ }^{24-27}$. A maior quantidade de prática de atividade física e de intensidade vigorosa expõem os indivíduos do sexo masculino a maior risco de lesões.

Indivíduos mais jovens apresentaram maior risco de lesão do que os de maior faixa etária, assim como demonstrado por Dempsey et al. ${ }^{8}$ Hootmann et al. ${ }^{22}$. Indivíduos mais jovens estão mais envolvidos com a prática de atividade física ${ }^{27}$, especialmente de esportes ${ }^{21}$, o que aumenta a possibilidade de lesionarem-se.

Sujeitos de cor da pele preta e parda estiveram em maior risco de lesões do que os de cor da pele branca. A relação entre cor da pele e lesões ainda vem sendo pouco estudada na literatura, particularmente considerando não atletas. As poucas pesquisas encontradas foram relacionadas a jovens praticantes de treinamento ou de esportes de alta intensidade. Estudo conduzido por Shafer et al. ${ }^{28}$ relatou falta de associação entre etnia e risco aumentado para fraturas durante treinamento físico intenso. Na mesma direção estão os estudos realizados por Laurino et al..$^{29}$ e Pileggi et al..$^{30} \mathrm{com}$ atletas de atletismo de ambos os sexos, mostraram não existir diferença no acontecimento de lesões entre os indivíduos de cor da pele preta e branca.

Apesar do desfecho do presente estudo considerar as lesões ocorridas durante a prática de AF e os nossos resultados terem indicado que as pessoas ativas estejam em maior risco para a ocorrência de lesões, devemos salientar, do ponto de vista da saúde pública, que não devemos deixar de praticar atividades físicas, pois os benefícios decorrentes do estilo de vida ativo são maiores e mais duradouros do que um estilo de vida sedentário ${ }^{31,32}$

Um aspecto a ser destacado nesse estudo é que a amostra pode ser considerada representativa dos adultos de 20 a 69 anos de idade residentes em Pelotas, tendo em vista o alto percentual de indivíduos entrevistados, o processo de amostragem aleatório e em múltiplos estágios e o baixo índice de perdas e recusas. No entanto, devemos salientar que o estudo é passível de viés de memória, especificamente no tipo de lesão sofrida pelo indivíduo, uma vez que o mesmo pode ter sofrido mais de um tipo de lesão em diferentes etapas da prática de atividades físicas e somente lembrar-se daquela(s) que mais Ihes causou incômodo. Além disso, não foram identificadas a intensidade e o volume das atividades físicas praticadas.

\section{CONCLUSÃO}

Os indivíduos do sexo masculino, de menor faixa etária e de cor da pele não branca apresentaram maior risco de lesões em consequência da prática de atividades físicas no tempo de lazer. Embora a prevalência de lesões em consequência da prática seja importante, os benefícios decorrentes de um estilo de vida ativo superam os riscos.

Todos os autores declararam não haver qualquer potencial conflito de interesses referente a este artigo. 


\section{REFERÊNCIAS}

1. World Health Organization. Global Status Report on non-communicable diseases 2010. Geneva: World Health Organization; 2011

2. Hallal PC, Andersen LB, Bull FC, Guthold R, Haskell W, Ekelund U. Global physical activity levels: surveillance progress, pitfalls and prospects. Lancet. 2012; 380(9838):247-57.

3. Heath GW, Parra DC, Sarmiento OL, Andersen LB, Owen N, Goenka S, et al. Evidence-based intervention in physical activity: lessons from around the world. Lancet. 2012;380(9838):272-81.

4. World Health Organization. A guide for population-based approaches to increasing levels of physical activity: implementation of the WHO Global Strategy on Diet, Physical Activity and Health. Geneva: World Health Organization; 2007.

5. Ory M, Resnick B, Jordan PJ, Coday N, Riebe D, Garber CE, et al. Screening, safety and adverse events in physical activity interventions: collaborative experiences from the Behavior Change Consortium. Ann Behav Med. 2005; 29 (special supplement):20-8.

6. Marar M, Mcllvain NM, Fields SK, Comstock RD. Epidemiology of concussions among United States high school athletes in 20 sports. Am J Sports Med. 2012; 40(4):747-55

7. Morey MC, Sullivan Jr. RJ. Medical assessment for health advocacy and practical strategies for exercise initiation. Am J Prev Med. 2003;25(3):204-8

8. Dempsey RL, Layde PM, Laud PW, Guse CE, Hargarten SW. Incidence of sports and recreation related injuries resulting in hospitalization in Wisconsin in 2000. Inj Prev. 2005;11(2):91-6.

9. Cheng TL, Fields CB, Brenner RA, Wright JL, Lomax T, Scheidt PC. Sports injuries: an important cause of morbidity in urban youth. Pediatrics. 2000;105(3):1-6.

10. Dekker R, Kingma J, Groothof JW, Eisha WH, Ten Duls HJ. Measurement of severity of sports injuries: an epidemiological study. Clin Rehabil. 2000;14(6):651-6.

11. Stevenson MR, Hamer P, Finch CF, Elliot B, Kresnow M. Sport, age, and sex specific incidence of sports injuries Western Australia. J Sports Med. 2000; 34(3): 188-94.

12. Ribeiro RM, Vilaça F, Oliveira HU, Vieira LS, Silva AA. Prevalência de lesões no futebol em atletas jovens: estudo comparativo entre diferentes categorias. Rev Bras Educ Fis Esporte. 2007;21(3):189-94.

13. Pastre CM, Carvalho Filho G, Monteiro HL, Netto Junior J, Padovani CR. Lesões desportivas na elite do atletismo brasileiro: estudo a partir de morbidade referida. Rev Bras Med Esporte. 2005;11(1):43-7.

14. Moreira P, Gentil G, Oliveira C. Prevalência de lesões na temporada 2002 da seleção brasileira masculina de basquete. Rev Bras Med Esporte. 2003;9(5):258-62.

15. Grego LG, Monteiro HL, Padovani CR, Gonçalves A. Lesões na dança: estudo transversal híbrido em academias da cidade de Bauru-SP. Rev Bras Med Esporte. 1999; 5(2):47-54.

16. Siqueira FV, Facchini LA, Hallal PC. Epidemiology of physiotherapy utilization among adults and elderly Rev Saude Publica. 2005;39(4):663-8.

17. Associação Brasileira de Empresas de Pesquisa. Critério de classificação econômica Brasil. Disponível em http:// www.abep.org/codigosguias/CCEB2008 Base 2006 e 2007.pdf. 2006. Acesso em 25 de Agosto de 2010.18.
18. World Health Organization. Physical status: the use and interpretation of anthropometry. Report of a WHO Expert Committee. Geneva: World Health Organization; 1995. (WHO Technical Report Series, 854).

19. Finch C, Valuri G, Ozanne-Smith J. Sport and active recreation injuries in Australia: presentations evidence from emergency department. J Sports Med. 1998;32(3):220-5.

20. Conte M, Matiello Junior E, Chalita LVS, Gonçalves A. Exploração de fatores de risco de lesões desportivas entre universitários de educação física: estudo a partir de estudantes de Sorocaba/SP. Rev Bras Med Esporte. 2002;8(4):151-6

21. Arena SS, Carazzato JG. A relação entre o acompanhamento médico e a incidência de lesões esportivas em atletas jovens de São Paulo. Rev Bras Med Esporte. 2007;13(4):217-21.

22. Hootman MJ, Macera AC, Ainsworth EB, Martin M, Addy LC, Steven NB. Association among physical activity level, cardiorespiratory fitness, and risk of musculoskeletal injury. Am J Epidemiol. 2001;154(3):251-8.

23. Hallal PC, Reichert FF, Siqueira FV, Dumith SC, Bastos JP, Silva MC, et al. Correlates of leisure-time physical activity differ by body-mass-index status in Brazilian adults. J Phys Act Health. 2008; 5(4):571-8

24. Azevedo MR, Araujo CLP, Reichert FF, Siqueira FV, Silva MC, Hallal PC. Gender differences in leisure-time physical activity. Int J Public Health. 2007;52(1):8-15.

25. Del Duca GF, Rombaldi AJ, Knuth AG, Azevedo MR, Nahas MV, Hallal PC. Associação entre nível econômico e inatividade física em diferentes domínios. Rev Bras Ativ Fis Saude. 2009;14(2):123-31.

26. Siqueira FV, Facchini LA, Silveira DS, Piccini RX, Tomasi E, Hallal PC. Leisure-time physical activity among aduit and elderly individuals in Brazil: a countrywide analysis. J Phys Act Health. 2011;8(7):891-7.

27. Silva MC, Rombaldi AJ, Azevedo MR; Hallal PC. Participação atual e passada em academias de ginástica entre adultos: prevalência e fatores associados. Rev Bras Ativ Fis Saude. 2008;13(1):27-35.

28. Shaffer RA, Brodine SK, Almeida SA, Williams KM, Ronaghy S. Use of simple measures of physical activity to predict stress fractures in young men undergoing a rigorous physical training program. Am J Epidemiol. 1999;149(3):236-42.

29. Laurino CFS, Lopes AD, Mano KS, Cohen M, Abdalla RJ. Lesões músculo-esqueléticas no atletismo Rev Bras Ortop. 2000;35(9):364-8.

30. Pileggi P, Gualano B, Souza M, Caparbo VF, Pereira RMR, Pinto ALS, et al. Incidência e fatores de risco de lesões osteomioarticulares em corredores: um estudo de coorte prospectivo. Rev Bras Educ Fis Esporte. 2010;24(4):453-62

31. Warburton DER, Gledhill N, Jamnik VK, Bredin SSD, McKenzie DC, Stone J, et al. Evidence-based risk assessment and recommendations for physical activity clearance: consensus document 2011. App Physiol Nutr Metab. 2011;36(Suppl 1):S266-8

32. Warburton DER, Gledhill N, Jamnik VK Bredin SSD, McKenzie DC, Stone J, et al International launch of the PAR-Q+ and ePARmed-X. The physical activity readiness questionnaire (PAR-Q+) and electronic physical activity readiness medical examination (ePARmed-X+): summary of consensus panel recommendations. Health Fit J Can. 2011;4(2):26-37. 